Manuelle Medizin 2017 ·55:1-2

DOI 10.1007/s00337-017-0240-1

Online publiziert: 2. Februar 2017

๑) Springer Medizin Verlag Berlin 2017

CrossMark

Lothar Beyer

ÄrztehausMitte Jena, Jena, Deutschland

\title{
Symbiose Praxis und Wissenschaft
}

\section{Bestandsaufnahme}

Es erscheint also durchaus zweckmäßig, mit einer Bestandsaufnahme des Vorhandenen zu beginnen und existierende Lücken zu schließen.

Die vorhandenen Untersuchungsund Behandlungstechniken wurden recht gut gesammelt und kategorisiert. $\mathrm{Ob}$ sie schon so klar klassifiziert sind, dass sie in das Gesamtsystem der universitären Medizin integriert werden können, wäre zu überdenken.

Welche Symptome, Krankheitszeichen, Syndrome, Funktionskrankheiten, Krankheitsbilder versorgen wir in der Praxis bei unseren Patienten? Haben wir davon ausreichend Zahlen, die unser Können belegen und die Potenz der MM aufzeigen? In den ANOA-Kliniken hat man als Erstes begonnen, dies zu erfassen und zu systematisieren, um aufzuzeigen, dass eine konservative manualmedizinisch-orthopädische stationäre Behandlung nicht nur einen Benefit für den Patienten bedeutet, sondern sich auch ökonomisch lohnen kann. Eine solche Bestandsaufnahme ist aber auch ungeheuer wichtig für die Konsensfindung unter den einzelnen Schulmeinungen, auch für das Finden von Fragestellungen, wo sich die MM auch im eigenen Interesse an der Versorgungsforschung beteiligen sollte. Dabei geht es nicht nur darum, welche Krankheitsbilder versorgt werden, sondern auch um die Inzidenz der in der manualmedizinischen Praxis erstellten Befunde und Diagnosen. Ein breites Feld, an dem sich aber jede Praxis mehr oder weniger beteiligen kann.

\section{Forschungsfelder bestimmen}

Abgeleitet aus den Ergebnissen der Bestandsaufnahme in der MM, insbesondere aber auch aus dem reichen praktischen Erfahrungsschatz der MM müssen wir aktuelle und perspektivische Forschungsfelder ableiten, wenn wir die $\mathrm{MM}$ als eigenständigen medizinischen Wissenschaftsbereich erhalten wollen und um gleichzeitig die MM weiter in die anderen medizinischen Fachgebiete zu integrieren. Janda, Vojta, Brügger, Lewit $u$. a. haben Theorien entwickelt, nach denen die manuelle Befunderhebung erfolgt und Therapiepläne erstellt werden. $\mathrm{Zu}$ wenig wurden aber bisher Fragestellungen daraus als zu überprüfende Hypothesen formuliert. Noch weniger wurden bestehende Fragestellungen überprüft, obwohl die einzelnen Lehrbücher solche Hypothesen ausreichend aufwerfen, die aus den in der Praxis gesammelten Daten hervorgegangen sind.

\section{Lösungsinstrumentarien schaffen}

Qualifizierte „man power" ist unabdingbar für die manualmedizinische Praxis. Man kann einschätzen, dass diese in den letzten Jahren herangebildet wurde und auch dafür weiterhin gute Voraussetzungen bestehen. Qualifizierte „man power" ist aber genauso unabdingbar für die Forschung in der MM: bereits bei der Bestimmung der Forschungsfelder und mehr noch bei der Lösung der Fragestellungen. Neugier und Leidenschaft Einzelner sind gefragt, aber eben auch Zeit für die Forschung und finanzielle Unterstützung. Unsere Potenzen sind hier schwach. Es haben sich aber bereits Interessengruppen gebildet, die Hoffnung keimen las- 
sen, dass wir auch auf wissenschaftlichem Gebiet Fortschritte machen können. Zu nennen sind hier die Forschungsgruppe der ANOA-Kliniken, der Arbeitskreis CMD in der Kieferorthopädie, der Zirkel für Manuelle Medizin und Entwicklungstherapie (ZIMMT) und die International Academy of Manual/Musculoskeletal Medicine(IAMMM). Diese Kräfte sollten sich zusammenschließen und Netzwerke bilden, die sich mit akademischen Einrichtungen verlinken. Solche akademischen Einrichtungen haben, wenn sie angewandte Forschung verfolgen, nicht selten Schnittmengen bei ihren Forschungsgegenständen mit der MM, so z. B. in der Arbeits- und Sportmedizin. Gelingt es uns nicht, solche Netzwerke zu knüpfen und gemeinsam interessierende Fragen $\mathrm{zu}$ bearbeiten, besteht durchaus die Gefahr, dass die Felder der MM von anderen besetzt werden. Die Folgen einer solchen Entwicklung wären ein Rückschritt für die MM. Warum sollten wir nicht in Richtung eines Instituts für MM denken und dieses evtl. auch mit einer Stiftungsprofessur besetzen? Hier sei noch an die jüngst gegründete Deutsche Stiftung Manuelle Medizin (www.stiftungmm.de) erinnert, über die ein erforderliches Fundraising angedacht ist.

Wir sind am Anfang des Jahres 2017, es bleibt also durchaus genügend Zeit, in diesem Jahr über die Symbiose von Praxis und Forschung in der MM nachzudenken und Folgerungen abzuleiten wir für uns alle.

Mit freundlichen Grüßen

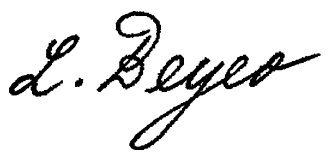

Lothar Beyer

\section{Korrespondenzadresse}

Prof. L. Beyer

ÄrztehausMitte Jena

Westbahnhofstr. 2, 07745 Jena, Deutschland

lobeyer@t-online.de

Interessenkonflikt. L. Beyer gibt an, dass kein Interessenkonflikt besteht.

\section{Jahrestagung der Vereinigung Süddeutscher Orthopäden und Unfallchirurgen e.V.}

\section{7. bis 29. April in Baden-Baden}

Getreu dem Kongressmotto „Das Ganze ist mehr...” werden die Kongresspräsidenten, Prof. Dr. Grützner und Prof.

Dr. Parsch, u.a. über den eigenen Tellerrand hinausschauen und haben deshalb Themen aus den „Grenzgebieten von O\&U“ mit in das Programm aufgenommen.

Insgesamt werden etwa 400 Programm-

punkte aus folgenden Schwerpunktthemen angeboten:

$$
\begin{aligned}
& \text { - Gelenkverletzungen } \\
& \text { - Endoprothetik } \\
& \text { - Wirbelsäule } \\
& \text { - Sportorthopädie } \\
& \text { - Praxis und Klinik } \\
& \text { - Freie Themen }
\end{aligned}
$$

Sehr wichtig ist den beiden Kongresspräsidenten der kollegiale Austausch. Sie betonen das einzigartige Gesamtpaket des Kongresses. „Es werden nicht nur Spezialisten für ein Gebiet angesprochen, sondern alle Orthopäden und Unfallchirurgen: Der niedergelassene Kollege genauso wie der Klinikarzt, der Anfänger wie der Fortgeschrittene".

Die Sitzungen Meine wichtigsten Fälle auf dem Weg zum guten ... (Kinderorthopäden, Tumorchirurgen, Endoprothetiker, etc.) sind ein Novum. Hier präsentieren ausgewiesene Experten ihre relevanten Fälle und den daraus abgeleiteten Erkenntnisgewinn.

Spannende Themen aus Grenzgebieten von O\&U werden in den zwei Sitzungsblöcken Das Ganze ist mehr... beleuchtet.

Die Podiumsdiskussion Pay for Performance mit Vertretern aus Politik, Klinik \& Praxis sowie den Krankenkassen soll mehr Transparenz und ein besseres Verständnis für das kontrovers diskutierte „P4P-Prinzip“ bringen.

Dr. Flechtenmacher (BVOU Berufsverband der Orthopäden und Unfallchirurgen) moderiert die Podiumsdiskussion Perspektiven für Klinik und Praxis in O\&U - wie geht es weiter? Fachkundige Repräsentanten nehmen zu den Fragen Arbeitsbedingungen, Sektorendefinition, Weiterbildung und Honorierungssystem in Klinik und Praxis Stellung.

\section{The Next Generation}

Der Nachwuchs garantiert die Zukunft in O\&U und ist seit Jahren ein wichtiges Thema auf dem VSOU-Kongress. In der Podiumsdiskussion Klinik der Zukunft @ Next Generation wird das Junge Forum O\&U gemeinsam mit den Nachwuchskräften Lösungen zu den notwendigen Veränderungen in Personalpolitik und Infrastruktur erarbeiten.

Auch 2017 wird es die Möglichkeit geben, praktische Fertigkeiten zu trainieren und Tricks von Profis zu lernen. Um allen Facetten des Faches O\&U gerecht zu werden, findet ein AE-Basiskurs und ein AO-Workshop statt. Die Teilnehmer können die Prinzipien von Reposition und Osteosynthese in konzentrierter Form erlernen bzw. sich mit den theoretischen und praktischen Grundlagen der Hüft- und Knieendoprothetik vertraut machen.

Erneut richtet die VSOU in Zusammenarbeit mit dem Jungen Forum und den YOUngster's O\&U einen Tag der Vorklinik aus.

\section{„Das Ganze und das Mehr"}

Zur Vollendung des Ganzen gehört auch der gesellige Austausch unter Kollegen und Freunden. Auf der Eröffnungsveranstaltung wird der bekannte Kabarettist Lars Reichow Das Ganze und das Mehr auf humorvolle und tiefgründige Art und Weise beleuchten.

Weitere Informationen unter: http://jahrestagung2017.vsou.de

\section{Organisation und Kongressleitung}

Geschäftsstelle der

Vereinigung Süddeutscher Orthopäden und Unfallchirurgen e. V.

Maria-Viktoria-Straße 9

D-76530 Baden-Baden

Tel.: +49 (0) $7221 / 29683$

info@vsou.de 